\title{
Investigation of the biofuel flue and producer gases cleaning efficiency using ESP
}

\author{
Robertas Poškas ${ }^{1, *}$, Arūnas Sirvydas ${ }^{1}$, Povilas Poškas $^{1}$, Nerijus Striūgas $^{2}$, Nerijus Pedišius $^{3}$, \\ and Vitas Valinčius ${ }^{4}$ \\ ${ }^{1}$ Lithuanian Energy Institute, Nuclear Engineering Laboratory, Breslaujos 3, Kaunas LT44403, \\ Lithuania \\ ${ }^{2}$ Lithuanian Energy Institute, Laboratory of Combustion Processes, Breslaujos 3, Kaunas LT44403, \\ Lithuania \\ ${ }^{3}$ Lithuanian Energy Institute, Laboratory of Heat Equipment Research And Testing, Breslaujos 3, \\ Kaunas LT44403, Lithuania \\ ${ }^{4}$ Lithuanian Energy Institute, Plasma Processing Laboratory, Breslaujos 3, Kaunas LT44403, \\ Lithuania
}

\begin{abstract}
The use of biofuel has been increasing in Europe over the last years, and the reason for that is acceptable cost and the least negative impact on the environment. However, $\mathrm{NO}_{\mathrm{x}}$ and emissions of fine particulates are important, and biofuel is still a disadvantage compared to oil and natural gas fired systems. Usually, flue gas is filtered in multicyclones or fibre filters before discharge into the atmosphere. Yet, in the case of fine particulates, the filters of such type do not show high effectiveness, thus electrostatic precipitators are used. In this comparative study on biofuel (wood pellets), the collection efficiency of solid particles from a class 3 boiler $(50 \mathrm{~kW})$ and from a gasification unit $(100 \mathrm{~kW})$ was investigated. Although releases of solid particles from modern boilers are low, a combination of such a boiler with an electrostatic precipitator may reduce the releases of particles to the minimum, and the collection efficiency of the electrostatic precipitator obtained during the investigation was $\sim 98-99 \%$. There is a big difference in particle concentrations comparing the systems with flue gas and producer gas. As the working conditions in the test section with producer gas were harder, it led to lower efficiency of the electrostatic precipitator $(\sim 75 \%)$.
\end{abstract}

\section{Introduction}

During the last decades, biofuel has been started to be widely used in many countries for various types of heating appliances. Biofuel is also a renewable energy source that makes the least negative impact on the environment in comparison with some other types of fuel [1]. During combustion of any liquid or solid fuel, particles are formed from the ash contained in the fuel. This is also valid for biofuels where the ash content varies from a fraction of a percent to more than ten percent by weight in the dry substance. Some of the

\footnotetext{
*Corresponding author: Robertas.Poskas@1ei.lt
} 
ash usually deposits in the furnace, whilst another part is carried out as cinder with the flue gas into the atmosphere [2]. Therefore, one of the main disadvantages using biofuel in comparison with some other types of fuels (gaseous, liquid) are the emissions of solid particles, which especially increase in the ambient air during the heating season. Solid particles can penetrate into the internal organs of human and can cause serious illnesses [3].

Since environmental protection problems are becoming more relevant, the requirements for production of small combustion appliances (with a nominal heat output up to $500 \mathrm{~kW}$ ) are being coordinated by EN303-5:2012 standard. In general, this standard aims to increase the efficiency of boilers and to reduce the emissions. It also indicates the limiting emission values of $\mathrm{SO}_{2}, \mathrm{NO}_{\mathrm{x}}, \mathrm{CO}$, organic gaseous carbon and solid particles depending on the boiler class.

Usually, before discharge into the atmosphere, flue gas is filtered (cleaned) in multicyclones or fibre filters. However, such types of filters are not very effective in the case of fine or ultrafine particulates; therefore, for that purpose electrostatic precipitators (ESPs) are used [4]. The advantages of electrostatic precipitators are low aerodynamic resistance and high efficiency [5]. Therefore, electrostatic precipitators are one of the most commonly employed particulate control devices for collecting particulate emissions from boilers, incinerators and from many other industrial installations.

In this comparative study on biofuel (wood pellets), the collection efficiency of solid particles from a European standard class 3 boiler $(50 \mathrm{~kW}$ nominal capacity) and from a gasification unit (100 $\mathrm{kW}$ nominal capacity) using an electrostatic precipitator is investigated.

\section{Experimental setups}

\subsection{Experimental setup with boiler}

Flue gas (solid particles) was generated by incinerating wood pellets in the boiler. The commercial boiler of class 3 was used. It is an automatic device with a nominal power of $50 \mathrm{~kW}$ and can be used for incineration of pellets, wood, pea-coal, and cereals (Fig. 1).

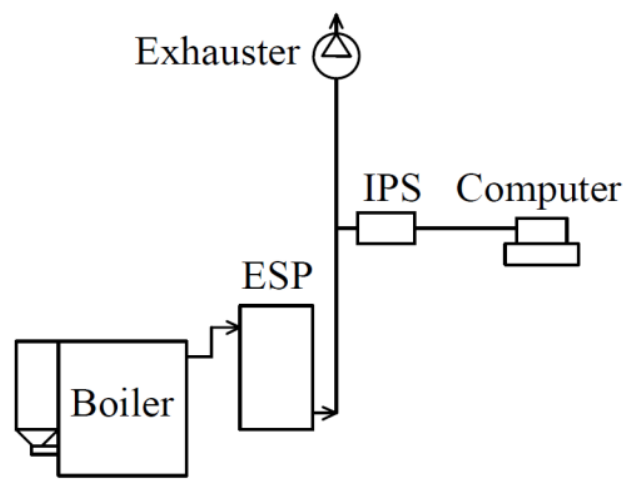

Fig. 1. Schematic view of the experimental setup with the boiler.

The fuel and air supply for combustion are adjusted automatically basing on the set power of the boiler. During the experiments, only wood pellets made from different kinds of wood were incinerated. Flue gas temperature exhausted from the boiler was about $150^{\circ} \mathrm{C}$, and the chimney draft $\sim 25 \mathrm{~Pa}$ (automatically kept by the flue gas exhauster). Such draft gives a flue gas velocity of approx. $1.9 \mathrm{~m} / \mathrm{s}$ in the flue gas pipe of $180 \mathrm{~mm}$ diameter (flow rate $\sim 180 \mathrm{~m}^{3} / \mathrm{h}$ ). 
From the boiler, flue gas enters the ESP, where the solid particles are captured and then cleaned flue gas is exhausted into the atmosphere. The total height of the insulated flue gas pipe was about $15 \mathrm{~m}$. The discharges of flue gas into the atmosphere are always at some distance from the boiler, and this section is the most important for measurement. Therefore, for particle measurements using an infrared particle sizer (IPS), the distance of $\sim 8 \mathrm{~m}$ from the ESP was selected. The measurement results were further processed using a computer.

\subsection{Experimental setup with gasification unit}

The main components of this experimental setup were the gasification unit (nominal power $100 \mathrm{~kW}$ ), the plasma unit, the gas cooler, the electrostatic precipitator and the boiler (Fig. 2).

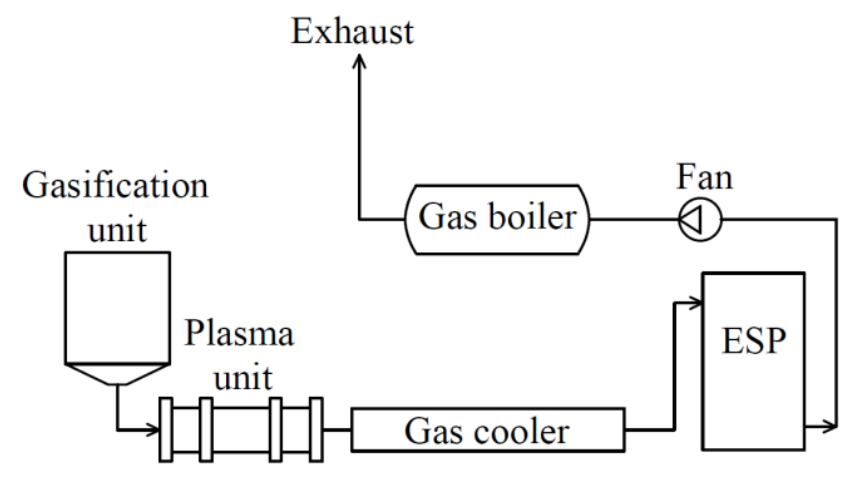

Fig. 2. Schematic view of the experimental setup with the gasification unit.

The process of wood pellets gasification (as well as generation of solid particles) took place in the gasification unit. The producer gas (flow rate $\sim 62 \mathrm{~m}^{3} / \mathrm{h}$ ) with a temperature of about $550^{\circ} \mathrm{C}$ entered the air plasma unit, which is needed for the neutralization of hazardous chemicals (dioxins, furans, etc. [6]), and existed as generated producer gas. The processed hot producer gas of about $1100^{\circ} \mathrm{C}$ from the plasma unit was supplied to the water-cooled gas cooler, where the temperature of the gas was decreased, and the gas with a temperature of $\sim 160^{\circ} \mathrm{C}$ entered the electrostatic precipitator. The cooled gas further flows via the electrostatic precipitator where the solid particles were captured from the gas flow. After passing the ESP, cleaned gas was transported by a fan to the gas boiler, and prepared flammable gas-air mixture was combusted. A more detailed description of the experimental setup is presented in [6].

The concentration of solid particles during these experiments was measured at the same time in the points before and after the ESP by applying the gravimetric method only.

\subsection{Electrostatic precipitator}

In the experiments with the boiler and the gasification unit was the same precipitator (see Fig. 3) used, and it was designed at the Lithuanian Energy Institute. Its frame is made from carbon steel bars. The inlet to the ESP is made in the upper part, and the outlet is positioned in the lower part. Inside the frame, two parallel stainless steel pipes (i.e. collection electrodes) with a diameter of $120 \mathrm{~mm}$ (each) and a length of $\sim 1000 \mathrm{~mm}$ are installed between two holding planes. A single nichrome wire (i.e. discharge electrode) of $0.2 \mathrm{~mm}$ is stretched between the special holders inside the center of each stainless steel pipe. The distance between the discharge electrodes is about $160 \mathrm{~mm}$. The tension of the electrodes is ensured by stainless steel springs. 
In order to avoid flue gas leakages between the holding planes and the collecting electrodes, the contact perimeters between them are sealed.

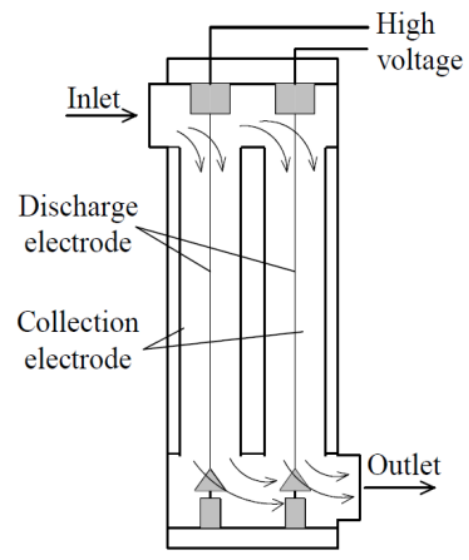

Fig. 3. Schematic cross-section view of the ESP used in the experiments.

During the experiments each of the discharge electrodes was connected to separate high-voltage power supply units (Glassman Series FR) with adjustable output voltage in the range between $0-30 \mathrm{kV}$. So, in total two high-voltage supply units were used. A more detailed description of the ESP is presented in [7]. The experiments were carried out using the negative potential of the high-voltage power supply units.

To reveal the peculiarities of the collecting efficiency of the ESP, different number of collecting electrodes ( 1 or 2$)$ were used. This allowed having different flow velocities. When one collecting electrode was used, the other pipe with the electrode was closed using special plates, and flue gas could flow only via the open pipe.

The current-voltage characteristic (high-voltage supply units used in the experiments had a feature enabling them to measure the current in an electrode depending on the supplied voltage) of one ESP electrode is shown in Fig. 4.

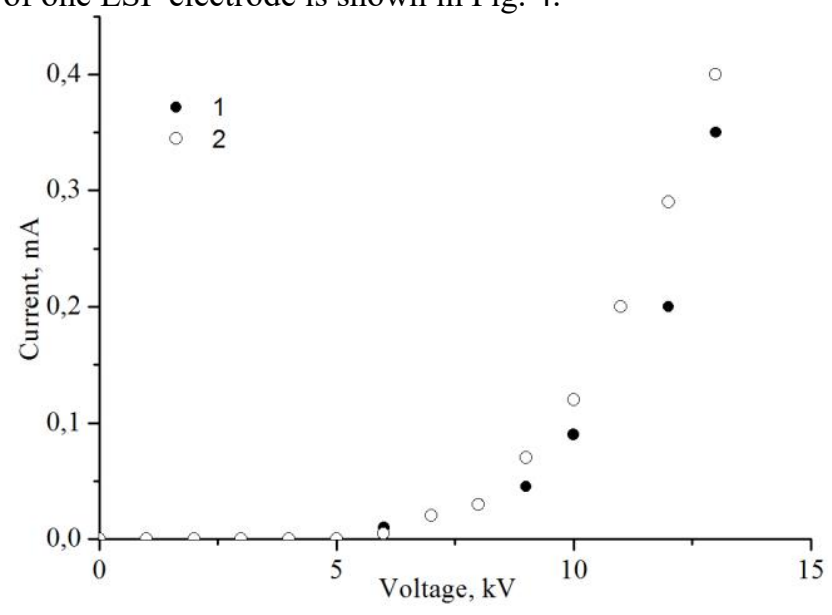

Fig. 4. Current-voltage characteristic of one ESP electrode: 1 - experimental data with the flue gas from the boiler; 2 - experimental data with the producer gas.

From the Figure it is evident that for flue gas from the boiler and for producer gas, the current is registered starting from the voltage of $\sim 6 \mathrm{kV}$. This means that from this point the electrostatic discharge, which ionizes the flue gas around the discharge electrode (i.e. 
creates corona), occurs. Although the character of the experimental data is rather similar in both cases, the layout of the experimental data is steeper for producer gas compared to flue gas from the boiler.

\subsection{Measurement techniques}

The concentrations of particles during the experiments with flue gas from the boiler were measured using the infrared particle sizer (IPS, made by "Kamika instruments") and applying the gravimetric method. The IPS is an instrument for direct measurements of total particle concentration and concentration of particle fractions in flue gas channels. It is composed of a measuring head and an electronic computer-controlled measurement unit. An IPS sizer ensures only 1D measurements and can measure particles in the range of $0.4-300 \mu \mathrm{m}$ (this range is divided into four subranges). The measuring head of the device is also equipped with a special holder for the filter, which can be used for the determination of concentration of solid particles by the gravimetric method. The IPS also measures flue gas temperature, concentration of solid particles, flue gas velocity and flow rate.

Distribution of particles by fraction in producer gas was not measured using the IPS because the gas was rather hot, humid and "dirty", and could foul the optical system of the device. Therefore, for producer gas only, the total concentrations of particles were measured before the inlet and after the outlet of the ESP using automatic isokinetic samplers Isostack Basic.

\section{Results and analysis}

Relative quantity of solid particle distribution in flue gas without the ESP is presented in Fig. 5.

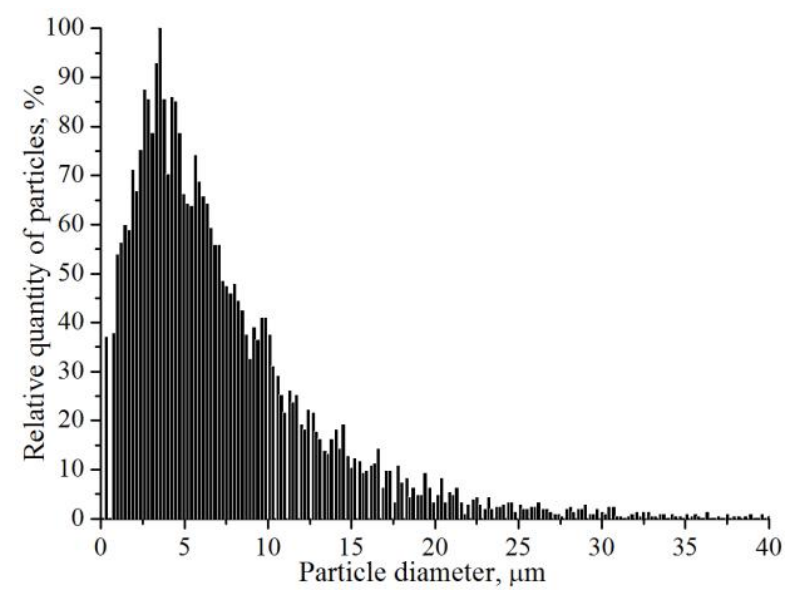

Fig. 5. Relative distribution of particles in flue gas without the ESP.

The Figure shows that flue gas mainly contains solid particles with diameters from 0.3 to $\sim 20 \mu \mathrm{m}$. The highest quantity of the particles in the distribution is at the diameter of about 2-6 $\mu \mathrm{m}$ (similar as presented in [8]).

Variation of the concentrations of solid particles during the steady-state experimental regime in producer gas and flue gas for the measurements during the experiments are presented in Fig. 6. Results obtained using the gravimetric method show that the initial concentration of solid particles in producer gas is rather high and is scattered in the range of $\sim 140-220 \mathrm{mg} / \mathrm{m}^{3}$, while the average value is about $180 \mathrm{mg} / \mathrm{m}^{3}$. 


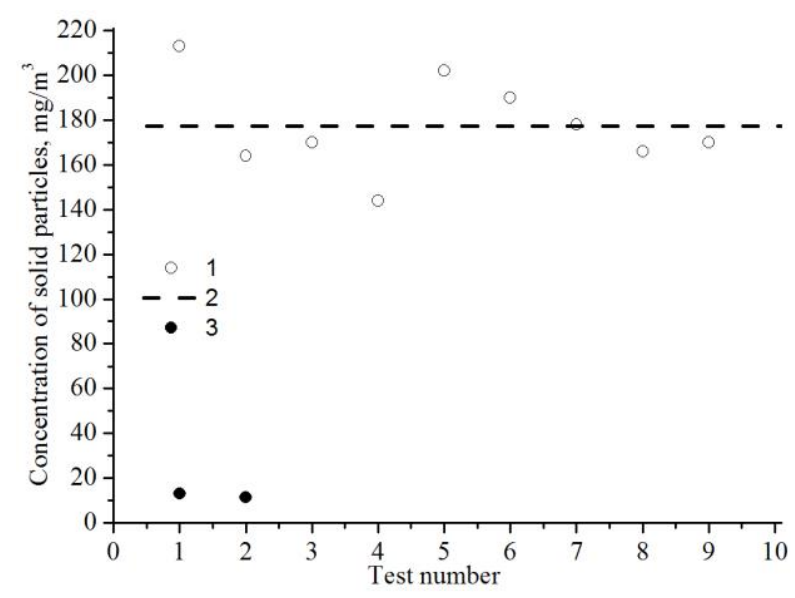

Fig. 6. Concentration of solid particles (for reference $\mathrm{O}_{2}$ value of $10 \%$ ) at the inlet to the ESP: 1 - in producer gas; 2 - average value in producer gas; 3 - in flue gas.

The concentration obtained for solid particles in flue gas is rather low - about $13 \mathrm{mg} / \mathrm{m}^{3}$. Similar results were obtained in [8] where particle concentrations from the modern $50 \mathrm{~kW}$ boilers fired by wood pellets vary in the range of $\sim 11-40 \mathrm{mg} / \mathrm{m}^{3}$. So, particle concentrations in flue gas are about fourteen times less in comparison with producer gas. The limiting values for such class of the boiler are much higher (cf. EN303-5:2012).

Total collection efficiency $E_{T i}$ presented in Fig. 7 was calculated using the equation:

$$
E_{T i}=\left(1-C_{i} / C_{o}\right) \cdot 100
$$

where $C_{o}$ is concentration of the particles obtained without the ESP; $C_{i}$ means concentration of the particles obtained with the ESP at a certain voltage.

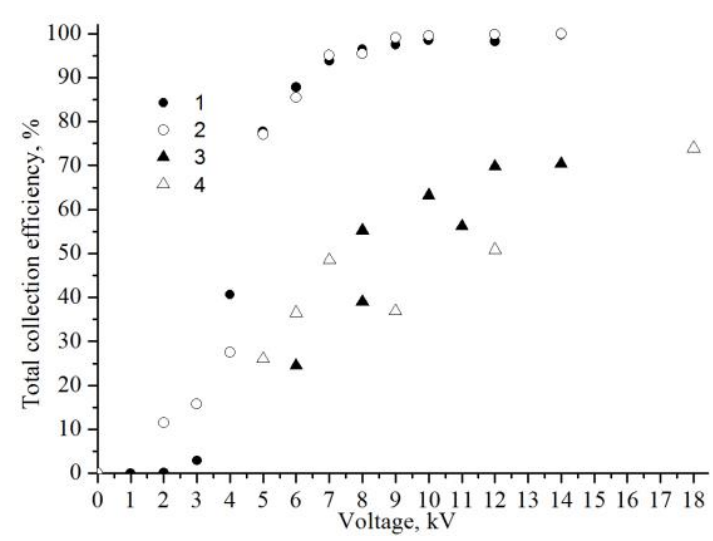

Fig. 7. Total collection efficiency of particles with the applied voltage to the ESP and in the case of a different number of collection electrodes: 1 - one collection electrode (flue gas); 2 - two collection electrodes (flue gas); 3 - one collection electrode (producer gas); 4 - two collection electrodes (producer gas).

The results with the flue gas show that in the region of voltages till $6 \mathrm{kV}$ (corona discharge registered), the collection efficiency just slightly depends on the number of used collection electrodes. 
From the voltage equal to or higher than $6 \mathrm{kV}$, the collection efficiency sharply increases and does not depend on the number of used collecting electrodes. The collection efficiency increases from about $75 \%$ at $6 \mathrm{kV}$ to about $98-99 \%$ at $14 \mathrm{kV}$. A further increase of the voltage does not give any tangible increase of the total collection efficiency.

For the case with producer gas, the dependency on used collection electrodes is not evidently expressed in the whole range of the applied voltages $(5-18 \mathrm{kV})$ either. Due to variations of the initial concentration of solid particles (Fig. 6), the collection efficiency in the case of one or two collecting electrodes is slightly scattered. With the increase of voltage, the collection efficiency is gradually increasing, and there is no sudden increase in the efficiency as it was observed for flue gas. For producer gas, the highest reached efficiency is only about $75 \%$ at a voltage of $18 \mathrm{kV}$. Further increase of the voltage does not increase the collection efficiency. This is due to the fact that the working conditions in the test section with producer gas is harder and less stable in comparison with fuel combustion in the boiler and therefore leads to lower collection efficiency of the electrostatic precipitator.

\section{Conclusions}

After the investigation of the biofuel flue from a class 3 boiler and producer gas cleaning efficiency using an ESP, the following conclusions have been drawn:

- The initial concentration of solid particles in flue gas was about $13 \mathrm{mg} / \mathrm{m}^{3}$ and about $180 \mathrm{mg} / \mathrm{m}^{3}$ in producer gas.

- The collection efficiency of the ESP used in the experiments practically did not depend on the number of used collection electrodes.

- At the voltage of the corona discharge, a sharp increase of the collection efficiency for solid particles in flue gas is observed, and further increase of the voltage gave only a slight increase of the collection efficiency of the ESP.

- At the voltage of the corona discharge, no sharp increase of the collection efficiency for solid particles in producer gas was observed; however, further increase of the voltage gave a gradual increase of the collection efficiency.

- The maximal total collection efficiency of the ESP was about 98-99\% for flue gas and about $75 \%$ for producer gas.

\section{References}

1. AEBIOM Stat. Rep. (2015)

2. J. Pettersson, M. Strand, L. Lin, Charging- and removal efficiency of an ESP in a $250 \mathrm{~kW}$ biomass boiler (ICESP, Nürnberg, 2011)

3. J.T. Zelikoff, L.C. Chen, M. Cohen, K. Fang, T. Gordon, Y. Li, C. Nadziejko, R.B. Schlesinger, Inh. Toxic 15, 131-150 (2003)

4. K.L. Parker, Applied electrostatic precipitation (Springer, Netherlands, 1997)

5. A.A. Varonos, J.S. Anagnostopoulos, G.C. Bergeles, J. Electr. 55, 111-133 (2002)

6. Z. Kavaliauskas, V. Valincius, G. Stravinskas, M. Milieska, N. Striugas, J. Air \& Wast. Manag. 65, 1292-1296 (2015)

7. R. Poskas, A. Sirvydas, P. Poskas, V. Simonis, J. Jankauskas, Mechan. (to be published)

8. N. Pedisius, M. Praspaliauskas, M. Valantinavicius, T. Vonzodas, V. Zaleskas, E. Lemanas, D. Cepauskienè, Pow. Eng. 62, 268-281 (2016) 